\title{
An Experimental Study on the Virtual Representation of Children
}

\author{
Ranchida Khantong * \\ Department of Computer Science \\ University College London
}

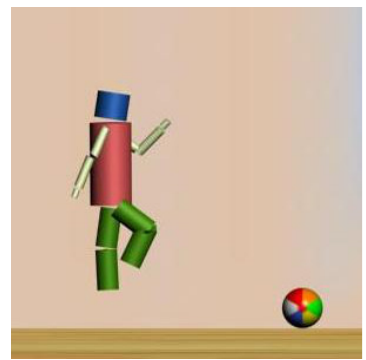

(a)

\author{
Xueni Pan \\ Institute of Cognitive Neuroscience \\ University College London
}

\author{
Mel Slater ${ }^{+}$ \\ UCL and ICREA \\ University College London
}

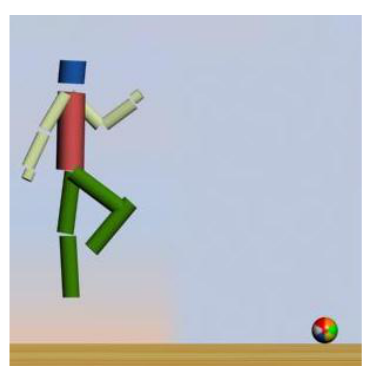

(b)

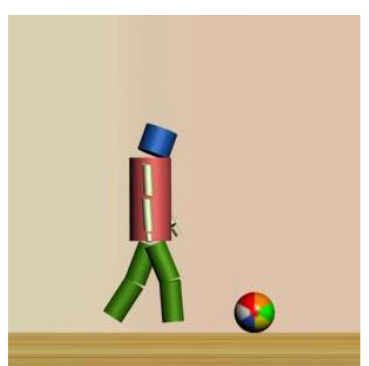

(c)

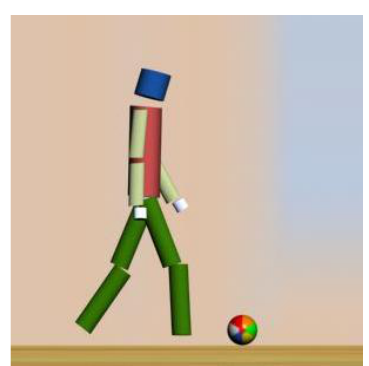

(d)

Figure 1: (a) $\mathrm{CaCb}$ : Child Animation with Child Body (b) CaAb: Child Animation with Adult Body (c) AaCb: Adult Animation with Child Body and (d) AaAb: Adult Animation with Adult Body.

\begin{abstract}
Is it their movements or appearance that helps us to identify a child as a child? In this work we created four video clips with a Virtual Character walking, but with different combinations of either child or adult animation applied on either a child or adult body. An experimental study was conducted with 53 participants who viewed all four videos in random orders. The results show that participants could easily identify the consistent video clips (child animation with child body, and adult animation with adult body). With the inconsistent video clips, both animation and body shape had an effect on participants' judgments. They also reported higher level of empathy, care, and feeling of protection towards the child character as compared to the adult character. Finally, compared to appearance, animation seems to be playing a bigger role in invoking participants' emotional responses.
\end{abstract}

Keywords: Virtual Characters, Computer Animation, User Study

\section{INTRODUCTION}

The virtual representation of children is more and more commonly seen in Virtual Reality applications, especially in computer games where virtual children normally appear as supporting characters. For example, in the game "Bioshock", as part of the plot players were continuously confronted with a dilemma of either to kill a child (the Little Sisters) to harvest a great deal of power, or to save the child to gain a smaller amount. The involvement of children is designed to provide an enhanced game play experience as they triggered sympathy in the player and could enhance the chance of a compelling gaming experience.

In this paper we discuss the implementation of a child character and a user study to validate our implementation: whether the "child character" is perceived as a "child", and whether it triggers a greater sympathetic reaction. Our second interest is the

*ucabrkh@ucl.ac.uk

$\dagger$ s.pan@cs.ucl.ac.uk

+m.slater@cs.ucl.ac.uk

IEEE Virtual Reality Conference 2015

23 - 27 March, Arles, France

978-1-4799-1727-3/15/\$31.00 @2015 IEEE interactive effect of animation and appearance. Four different video clips were generated, with different combinations between child/adult body and child/adult animation.

\section{BACKGROUND}

In everyday life it is easy to distinguish a child from an adult from both their appearance and behavior. The appearance of a child is obviously different from that of an adult, not only with respect to their sizes but also their body proportions - the body of a child is not a simple geometric scaling from an adult. Children are characterized with bigger heads and shorter limbs as compared to their torso: infants' lower limbs contribute only $15 \%$ of their whole body weights as compared to $30 \%$ for adults [1,2].

Because of the differences in their physical models, the movements of children are also different from adults. Moreover, as their brain is still developing, children have more variations in their movements and are clumsier as compared to adults [1,2]: for instance, instead of walking or running, children more often display movements such as jumping, hopping, skipping, climbing, and swinging. Another typical behavior that distinguishes them from adults is that when confronted with obstacles, children are more careful and require a larger clearance region for the lead foot to cross the obstacle [3]. There are also differences in movements of their upper body: children often use gesturing as part of their communication, and their gesturing movements are slower and jerkier than that of adults $[4,5]$. In general, the movements of children may be considered as more playful and more dramatic.

\section{ExPerimental Design}

In this study an abstract representation of a child was created using 3dsMax as shown in Fig 1(a). This is in order to eliminate unrelated variables (i.e., gender, ethnic issues), and is consistent with our current work on study of using VR in morality issues [6].

The child character (Fig. 1(a)) has a bigger head and shorter limbs as compared to the abstract model of an adult (Fig. 1(b)). Footstep animation was used to generate the basic walking movements but was then adjusted for the character to display skipping. Other child-like behaviors were also implemented, including playful behaviors such as jumping over obstacles and gesturing. The final animation was exported as a video clip which lasts 52 seconds. A corresponding animation was implemented with an adult walking through the same path and with the same 
length. Both child and adult animation were applied on both the child and the adult body and therefore generated 4 video clips: child animation with child body $(\mathrm{CaCb})$, child animation with adult body $(\mathrm{CaAb})$, adult animation with child body $(\mathrm{AaCb})$, and adult animation with adult body (AaAb). This was a two-factor within groups experiment. The two factors were Animation Type (child, adult) and Body Type (child, adult).

Overall, 53 participants attended our study: 15 females and 38 males (average age $=30 \pm 9.7$ S.D.). They were presented with all four videos in counter-balanced order. After watching each video clip, they were given a questionnaire that asked them to respond to that particular video. Finally after watching all four videos, they were asked to complete a further questionnaire about their personal information (e.g., age, gender).

\section{Results}

\subsection{Was the Virtual Character Recognized as a Child?}

TABLE 1: Proportion of participants who identified the $\mathrm{VC}$ as a Child for each video clip. $\mathrm{N}=53$

\begin{tabular}{|c|c|c|c|}
\hline \multicolumn{2}{|c|}{ Video Clip } & \multicolumn{2}{c|}{ Animation } \\
\cline { 3 - 4 } & Child & Child & Adult \\
\hline \multirow{2}{*}{ Body } & Adult & $56 \%$ & $36 \%$ \\
\cline { 2 - 4 } & Ad & $6 \%$ \\
\hline
\end{tabular}

Immediately after watching each video clip, participants were asked whether they recognized the VC in the scene as a child or adult. Table 1 shows the proportion of participants who identified the VC as a Child for each of the four conditions. As expected, most participants judged the first video (child animation + child body) as a child, and the least number of participants judged the fourth video (adult animation + adult body) as a child. With the responses as binomial (the number of participants who identified the character as a "child"), a repeated measure two-way ANOVA test shows that both Animation and Body $(\mathrm{p}<0.00001)$ has an effect on participants' judgments. There is no interaction effect. However the character was more likely to be judged as a child in $\mathrm{CaAb}$ as compared to $\mathrm{AaCb}$, which indicates animation might be playing a bigger role than appearance.

We also asked the participant to give an "estimated age" of the character. We asked participants to choose an age group in which the VC was most likely to be (1: $<5$ years; $2: 5-12$ years; $3: 12-18$ years; 4: $>18$ years). A repeated measure two-way ANOVA showed that both appearance and animation have a significant effect on age estimation $(\mathrm{P}<0.00001)$. Moreover, there is an interaction effect $(p<0.035)$ : as shown in Figure 2, when Child Body and Child Animation are together, the effect is more than the separate effect of each of them.

\subsection{Feelings toward the Virtual Character}

TABLE 2: Participants' feeling towards the VC. $\mathrm{N}=53$

\begin{tabular}{|c|c|c|c|}
\hline Feelings & Empathy & Care & Projection \\
\hline $\mathbf{C a C b}$ & $4.34 \pm 0.27$ & $4.34 \pm 0.27$ & $4.34 \pm 0.27$ \\
\hline $\mathbf{C a A b}$ & $4.13 \pm 0.25$ & $4.13 \pm 0.25$ & $4.13 \pm 0.25$ \\
\hline $\mathbf{A a C b}$ & $3.60 \pm 0.28$ & $3.60 \pm 0.28$ & $3.60 \pm 0.28$ \\
\hline $\mathbf{A a A b}$ & $3.11 \pm 0.28$ & $3.11 \pm 0.28$ & $3.11 \pm 0.28$ \\
\hline
\end{tabular}

We also asked participants for each video clip: (1) How much empathy they have towards the VC? (2) How much they care about the VC? and (3) How much they feel like protecting the VC? For each questions, participants could choose from 0 (not at all) to 8 (very much).

As shown in Table 2, the $\mathrm{CaCb}$ video triggered the highest level of empathy, care, and feeling of protection in the participants, with $\mathrm{AaAb}$ video the lowest. A repeated measure two-way ANOVA test shows that Animation has an effect on the level of empathy $(p<0.0001)$. Body also has an effect on empathy but with a lower significant level $(p<0.05)$. Both Animation and Body have an effect on level of care (Animation $p<0.00001$, Body $p<0.00001$ ), and feeling of protection (Animation $p<0.00001$, Body $p<0.00001$ ).

Also similarly to their judgment of the $\mathrm{VC}$ being a child or adult, for video $\mathrm{CaAb}$ and $\mathrm{AaCb}$ where there was an inconsistency in the video, participants seems to feel more empathy, care, and protection towards the video with child animation applied on an adult body as compared to the video with adult animation applied on a child body. Again, animation seems to have had the greater impact on participants' feelings.

\section{Means and 95\% Confidence Intervals for Each Group}

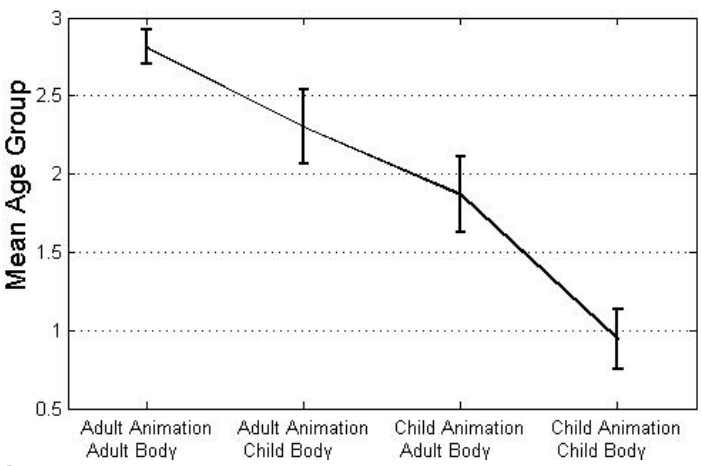

FIGURE 2: Estimated age group for each video clip (1: $<5$ years; 2: $5-12$ years; $3: 12-18$ years; $4:>18$ years).

\section{Conclusion}

First, the results validated the child representation (child body and animation) since the vast majority of participants successfully identified the consistent videos. Moreover, we found that with the consistent videos, the child character triggered significant higher level of sympathetic in the participants as compared to the adult character. These results form a useful basis for future studies that use child characters. Secondly, in this work we also discussed the impact of behavior and appearance in character representation. Our results suggest that when there is a conflict between animation of behavior and appearance, participants' judgments and emotional response were more likely to be dominated by the animation behavior rather than appearance.

\section{ACKNOWLEDGEMENT}

This research is funded by the Leverhulme Trust project "The exploitation of immersive virtual reality for the study of moral judgments".

\section{References}

[1] D. Morris and G. Desebrock, Manwatching: A field guide to human behaviour: HN Abrams New York, 1977.

[2] J. MacGregor, Introduction to the Anatomy and Physiology of Children: A Guide for Students of Nursing, Child Care and Health: Taylor \& Francis, 2008.

[3] J. R. Berard and L. A. Vallis, "Characteristics of single and double obstacle avoidance strategies: a comparison between adults and children," Experimental brain research, vol. 175, pp. 21-31, 2006.

[4] M. Argyle, Bodily communication: Methuen, 1988.

[5] N. E. Berthier and R. Keen, "Development of reaching in infancy," Experimental brain research, vol. 169, pp. 507-518, 2006.

[6] X. Pan and M. Slater, "Confronting a Moral Dilemma in Virtual Reality: A Pilot Study," BSC Human-Computer Interaction, 2011. 